\title{
MIMO-OFDM in a Cooperative Frequency Selective Fading Channel: An Analysis
}

\author{
Saurabh Dixit $\mathbb{D},{ }^{1}$ Himanshu Katiyar, ${ }^{2}$ and Arun Kumar Singh $\mathbb{D}^{3}$ \\ ${ }^{1}$ Department of Electronics, CIPET, Lucknow, India \\ ${ }^{2}$ Department of Electronics, REC, Sonebhadra, India \\ ${ }^{3}$ Department of Electronics, REC, Kannauj, India \\ Correspondence should be addressed to Saurabh Dixit; saurabh2911@ieee.org
}

Received 2 January 2019; Revised 8 June 2019; Accepted 17 September 2019; Published 3 November 2019

Academic Editor: Youyun Xu

Copyright (c) 2019 Saurabh Dixit et al. This is an open access article distributed under the Creative Commons Attribution License, which permits unrestricted use, distribution, and reproduction in any medium, provided the original work is properly cited.

Diversity is an integral technique in the advancement of wireless communications. Multiple-input multiple-output (MIMO) technology continues to occupy a pivotal position in the fourth-generation $(4 \mathrm{G})$ standards and beyond. With the advent of fulldimension (FD) MIMO and massive MIMO, new paradigms are being targeted in the disruptive technology. Third-generation partnership project (3GPP) has also emphasized the advanced MIMO technology for achieving its vision of International Mobile Telephony (IMT) 2020. However, implementing multiple antennas can be cumbersome due to limiting factors such as size, cost, and hardware limitations. In such a scenario, where implementing many antennas is not feasible, the benefits of diversity can be reaped by employing cooperative communication. Single antenna mobiles can share their mobile stations giving rise to a virtual MIMO system. In this paper, the authors have analyzed the performance of cooperative mobile stations in a frequency selective channel. Orthogonal frequency division multiplexing (OFDM), known for its robustness against multipath fading, is the modulation technique employed to circumvent the hazardous effects of intersymbol interference (ISI). MIMO is the key technology for achieving capacity advantage and improving link reliability. The blend of these two cutting edge technologies in a cooperative relay system presents encouraging results and inferences. Maximal Ratio Combining (MRC) is employed at the relay and the receiver to maximize the signal-to-noise ratio (SNR). Decode and forward (DF) protocol is used at the relay node owing to its regenerative nature and adaptability. The closed-form expressions for bit error rate (BER) and outage probability $\left(P_{\text {out }}\right)$ are derived and compared with the experimental results. The effect of power decay factor is analyzed and simulation is done for $2 \times 2$, $2 \times 3$, and $2 \times 4$ transmit and receive antennas. The number of antennas at the relay is kept as 2 . The analytical results agree with the simulation results.

\section{Introduction}

Wireless communications have exploited the diversity technique since its inception and continues to capitalize on its multiple advantages in beyond fourth-generation (4G) standards. With the advent of full-dimension (FD) MIMO and massive MIMO, new paradigms are being targeted in the disruptive technology. Third-generation partnership project (3GPP) has also emphasized the advanced MIMO technology for achieving its vision of International Mobile Telephony (IMT) 2020. However, implementing multiple antennas can be cumbersome due to limiting factors such as size, cost, and hardware limitations. In such a scenario, where implementing multiple antennas is not feasible, the benefits of diversity can be reaped by employing cooperative communication. Single antenna mobiles can share their mobile stations giving rise to a virtual MIMO system. In this paper, we have analyzed the performance of cooperative mobile stations in a frequency selective channel. Orthogonal frequency division multiplexing (OFDM), known for its robustness against multipath fading, is the modulation technique employed to circumvent the hazardous effects of intersymbol interference (ISI).

The concept of relaying was introduced by Van Der Meulen in 1971 [1]. Cover and Gamal [2] provided an insight into relay-based communication by analyzing the capacity of 
three node network, namely, the source, destination, and relay. The channel considered was additive white Gaussian noise (AWGN). Tarokh et al. in 1999 [3] introduced the concept of space-time block codes by the use of orthogonality. In their landmark paper, Gupta and Kumar in 2000 [4] have statistically analyzed the theoretically offered throughput for large-scale relay networks. Sendonaris et al. in 2003 [5] proposed the concept of implementing spatial diversity without using multiple antennas. The fundamental principle is to efficiently establish multiple antennas in a distributed manner by allowing neighboring nodes to share their radio resources. Space-time cooperation can be applied to a broad range of applications, such as broadcast networks, sensor networks, and relay networks. Nosratinia et al. in 2004 [6] have provided a vivid overview of cooperative communication technique. The authors opine that as long as the fading path is uncorrelated, the benefits of spatial diversity are reaped. The trade-off between total power consumed and sum rate is analyzed. A comparison of performance in cooperation signaling methods is provided. The results demonstrate that coded cooperation and decode and forward scheme perform better than without cooperation. Dohler et al. in 2003 [7] have introduced the concept of multihop distributed MIMO and deduced capacity advantages over SISO multihop communication. Laneman et al. in 2004 [8] have comprehensively evaluated the diversity order of multiple access protocols such as amplify and forward protocol and decode and forward protocol. The authors have demonstrated that cooperation yields full spatial diversity. Cui et al. in 2004 [9] have demonstrated that over certain distance ranges, the total energy consumption can be reduced. However, in short ranges, the performance of single-input single-output (SISO) may outperform multiple-input multiple-output (MIMO) or cooperative MIMO as far as energy efficiency is concerned. Shin et al. in 2007 [10] designed an OFDM spacetime cooperative system and achieved results demonstrating a significant performance advantage with cooperative OFDM. Tolli et al. in 2008 [11] have investigated the performance of nodes in a soft handover region, receiving a signal from distributed base stations cooperating amongst themselves. The authors opine that base station cooperation enhances the system complexity. Baek and Song in 2008 [12] have designed and analyzed the performance of cooperative diversity in MIMO-OFDMA system. The authors demonstrate that high-performance gains are achieved while using cooperative diversity. Jing and Jafarkhani in 2009 [13] have analyzed the performance of single and multiple relays and evaluated their diversity order.

The authors have focused on network relay selection schemes and propose suboptimal schemes with linear complexity. A generic cooperative relay model is depicted in Figure 1 . The mobile nodes cooperate mutually to enhance the signal-to-noise-ratio (SNR). Each mobile station or user collaborates with one another user or with a few users jointly and emulates a virtual MIMO methodology concept.

The protocols employed in cooperative communications can be classified into generative and transparent relaying protocols. In generative relaying protocols, the information

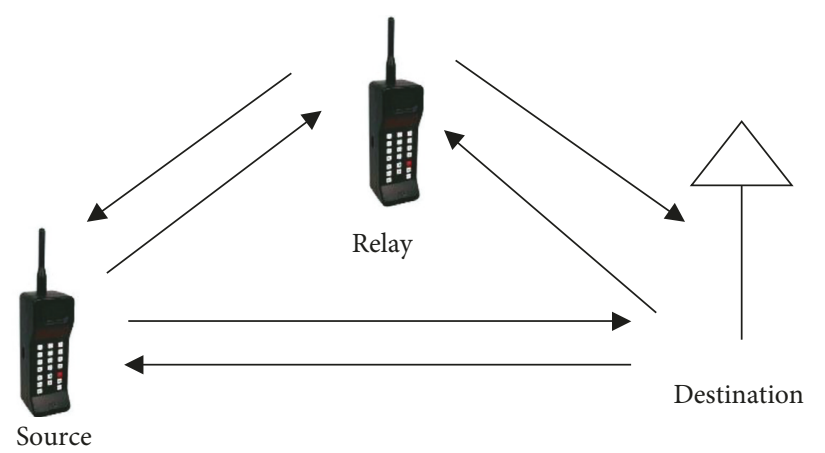

FIgUre 1: Cooperative relay model.

bits are modified and, based on a decision, relayed towards the destination. Decode and forward (DF) protocol is the commonly employed regenerative protocol, where the information bits are detected, decoded, and sent forward. This scheme has been shown to reduce channel interference and additive noise at the relay as demonstrated by $\mathrm{Lu}$ et al. in 2010 [14]. Furthermore, Hasna and Alouini in 2003 [15] demonstrated in their pioneering work that regenerative protocols perform better at low SNR. However, regenerative protocols require more complex baseband processing.

In the transparent relaying protocols, also known as nonregenerative protocols, there is no modification in the information bits. Power scaling and phase rotation are done at the relay before forwarding the signal. Amplify and forward (AF) protocol is the commonly employed nonregenerative protocol, where the information bits are amplified and sent forward. However, noise is also amplified and relayed in this protocol. Therefore, this protocol is not suitable for low SNR.

The key contributions of this paper are as follows:

(i) An OFDM-modulated MIMO relay channel with MRC at relay and destination is developed

(ii) Closed-form expressions for outage probability $\left(P_{\text {out }}\right)$ and bit error rate (BER) are evaluated

(iii) The simulation results and numerical results are evaluated for power decay factor of 0,1 , and 2 , respectively

(iv) The simulation and numerical results agree with each other, hence validating our model

(v) The signal-to-noise-ratio (SNR) is kept low to validate the efficacy of decode and forward protocol (DF) protocol

\section{System Model}

Figure 2 depicts our system model where OFDM-modulated signal are transmitted using multiple antennas. Chalise and Vandendorpe in 2008 [16] have developed a similar model for a Rayleigh flat-fading channel. There is a direct link between source and destination characterized by channel $h_{\mathrm{sd}}$. The channel between source and relay is defined as $h_{\mathrm{sr}}$. The channel between source and destination is $h_{\mathrm{sd}}$ while that between relay and destination is $h_{\mathrm{rd}}$. The communication 


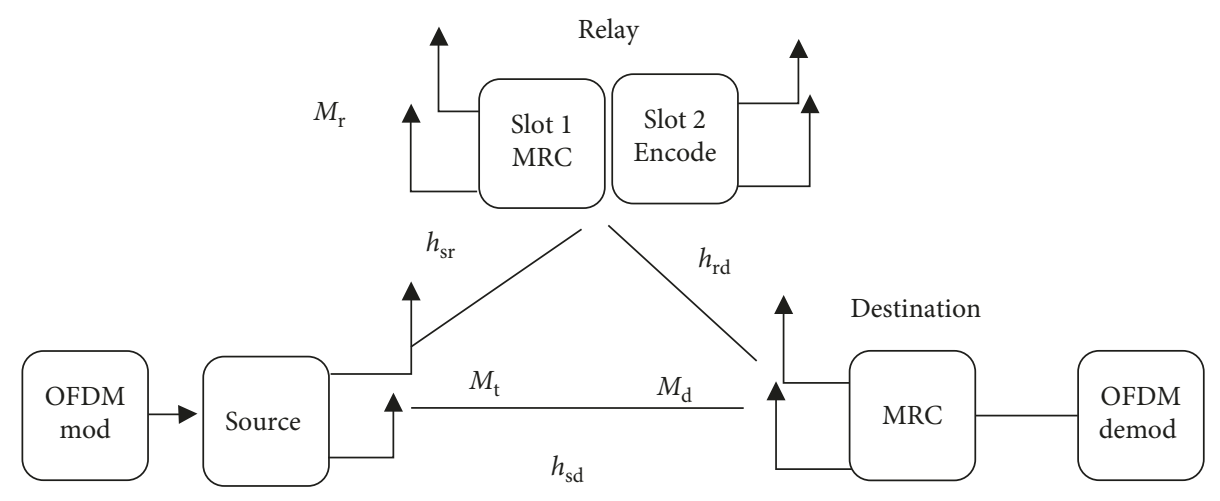

Figure 2: System model (MIMO-OFDM with relay nodes).

between source and destination occurs in orthogonal spacetime block coding (OSTBC). The following assumptions hold true in our model:

(i) The number of antennas at the source, relay, and destination are $M_{t}, M_{\mathrm{r}}$, and $M_{\mathrm{d}}$, respectively.

(ii) The channels $h_{\mathrm{sd}}, h_{\mathrm{sr}}$, and $h_{\text {rd }}$ are independent and identically distributed with zero mean and circularly symmetric complex Gaussian (ZMCSG).

(iii) The distance between source and destination is significant as compared to that between relay and destination.

(iv) The instantaneous SNR at the relay is greater than the threshold SNR required for relay mode to be active.

(v) In the first time slot, the source sends its orthogonal signals to relay and destination. The relay uses MRC and decodes the signal.

(vi) In the second time slot, the relay transmits the decoded signal to the destination which combines the signal of two time slots.

\section{Numerical Expression and Modeling}

Table 1 outlines the simulation parameters of the model. The number of antennas at the source and the relay is kept the same for uniform space-time coding. The antennas at the relay and destination perform MRC to enhance the SNR. Binary phase shift keying (BPSK) modulation is employed for simplicity. The channel is frequency selective in nature with two-channel taps with Rayleigh distribution.

\section{Mathematical Modeling}

We start our modeling by taking the probability density function (PDF) of a Rayleigh frequency selective channel defined in (1) as described by Dixit and Katiyar in 2018 [17]:

$$
p_{\gamma_{\mathrm{sr}}}(\gamma)=\frac{1}{4 \Omega_{\mathrm{sr}} S} e^{(-\gamma) / 4 \Omega_{\mathrm{sr}} S},
$$

where $p_{\gamma_{\mathrm{sr}}}(\gamma)$ is the PDF of SNR from source to relay, $\Omega_{\mathrm{sr}}$ is the sum of average channel power from the source to relay, $S$
TABLE 1: Simulation parameters of OFDM-MIMO relay channel.

\begin{tabular}{lc}
\hline Simulation parameter & Value \\
\hline $\begin{array}{l}\text { Number of transmit } \\
\text { antennas }\left(M_{t}\right)\end{array}$ & 2 \\
$\begin{array}{l}\text { Number of relay } \\
\text { antennas }\left(M_{\mathrm{r}}\right)\end{array}$ & 2 \\
$\begin{array}{l}\text { Number of destination } \\
\text { antennas }\left(M_{\mathrm{d}}\right)\end{array}$ & $2 / 3 / 4$ \\
Combining scheme & MRC \\
Modulation & BPSK \\
Number of channel taps & 2 \\
Channel & Frequency selective Rayleigh \\
Number of Monte Carlo & fading channel \\
simulation & $2 e 6$ \\
SNR $(\mathrm{dB})$ & -18 to $0 \mathrm{~dB}$ \\
\hline
\end{tabular}

is the ratio of Bit energy $\left(E_{\mathrm{b}}\right)$ to noise spectral density $\left(N_{\mathrm{o}}\right)$, and $\gamma$ is the instantaneous SNR. The PDF of SNR from the relay to the destination can be defined as follows:

$$
p_{\gamma_{\mathrm{rd}}}(\gamma)=\frac{1}{4 \Omega_{\mathrm{rd}} S} e^{(-\gamma) / 4 \Omega_{\mathrm{rd}} S},
$$

where $p_{\gamma_{\mathrm{rd}}}(\gamma)$ is the PDF of SNR from the relay to the destination, $\Omega_{\text {rd }}$ is the sum of average channel power from relay to the destination, $S$ is the ratio of Bit energy $\left(E_{\mathrm{b}}\right)$ to noise spectral density $\left(N_{\mathrm{o}}\right)$, and $\gamma$ is the instantaneous SNR. The cumulative distribution function (CDF) of the PDF is evaluated by its integral such that $P_{\mathrm{sr}}(\gamma)=1-e^{(-\gamma) / 4 \Omega_{\mathrm{sr}}}$; therefore, the CDF of the source to relay to the destination will be defined by (3) as done in Papoulis and Pillai in 2002 [18]:

$$
P_{\text {srd }}(\gamma)=1-\left[\left(1-P_{\gamma_{\text {sr }}}(\gamma)\right)\left(1-P_{\gamma_{\text {rd }}}(\gamma)\right)\right],
$$

where $P_{\text {srd }}(\gamma)$ is the CDF of source-relay-destination. For IID, $\gamma_{\mathrm{sr}}=\gamma_{\mathrm{rd}}=\gamma$. Therefore, the CDF in (3) can be simplified as

$$
P_{\text {srd }}(\gamma)=1-\left[\left(1-P_{\gamma}(\gamma)\right)^{n}\right]
$$

where $n=M_{t}=M_{\mathrm{r}}$ is the number of antennas at the relay. For $M=M_{\mathrm{d}}$ number of independent and identical fading antennas at the destination, the CDF in (4) is modified as follows: 


$$
P_{\text {srd }}(\gamma)=\left[1-\left\{1-P_{\gamma}(\gamma)\right\}^{n}\right]^{M}=\left[1-\left\{-e^{(-\gamma) / 4 \Omega S}\right\}^{n}\right]^{M}
$$

Upon differentiating (5), we obtain the PDF of SNR of the source to relay to the destination as follows:

$$
p_{\gamma_{\text {srd }}}(\gamma)=M\left[1-\left\{1-P_{\gamma}(\gamma)\right\}^{n}\right]^{M-1} \frac{\mathrm{d}}{\mathrm{d} \gamma}\left(1-\left\{-e^{(-\gamma) / 4 \Omega S}\right\}^{n}\right) .
$$

Upon differentiating (6), it can be solved as follows:

$$
\begin{aligned}
p_{\gamma_{\text {srd }}}(\gamma) & =M\left[1-\left\{1-P_{\gamma}(\gamma)\right\}^{n}\right]^{M-1 n\left(-e^{(-\Upsilon) / 4 \Omega S}\right)^{n}} \\
4 \Omega S & \\
& =\frac{M n}{4 \Omega S}\left[1-e^{-(\gamma n) / 4 \Omega S}\right]^{M-1}\left(-e^{-\gamma n / 4 \Omega S}\right) .
\end{aligned}
$$

To solve (7), we use the binomial relation

$$
\left(1-e^{-t}\right)^{M}=\sum_{k=0}^{M}{ }_{k}^{M}\left(-\mathrm{e}^{-t}\right)^{k}
$$

Thus, (7) can be written as

$$
p_{\gamma_{\text {srd }}}(\gamma)=\sum_{k=0}^{M-1} M-1 \quad\left(-e^{(-\gamma n) / 4 \Omega S}\right)^{k} \frac{M n}{4 \Omega S} e^{(-\gamma n) / 4 \Omega S}
$$

Therefore, the BER can be expressed as described by Goldsmith ([19], equation (6.50)):

$$
\begin{aligned}
\text { BER } & =\int_{0}^{\infty} P_{s}(\gamma) p_{\gamma_{\text {srd }}}(\gamma) \mathrm{d} \gamma \\
& =\sum_{k=0}^{M-1} M-1 \quad \frac{m n}{4 \Omega S} \int_{0}^{\infty} Q(\sqrt{2 \gamma})\left(-e^{-((-\gamma n k) / 4 \Omega S)}\right) e^{-(\gamma n) / 4 \Omega S} \mathrm{~d} \gamma,
\end{aligned}
$$

where $P_{\mathrm{s}}(\gamma)$ is the probability of error in additive white Gaussian noise (AWGN). To simplify (10), the identities in 5A.2 and 5A.3 in Simon and Alouini [20] are employed, as illustrated in (11) and (12):

$$
\begin{aligned}
J_{m}(a, b) \triangleq & \frac{a^{m}}{\Gamma(m)} \int_{0}^{\infty} e^{-a t} t^{m-1} Q(\sqrt{2 \gamma}) \mathrm{d} \gamma \\
J_{m}(a, b) \triangleq & J_{m}(c)=\frac{\sqrt{c / \pi}}{2(1+c)^{m+(1 / 2)}} \\
& \cdot \frac{\Gamma(m+(1 / 2))}{\Gamma(m+1)}{ }_{2} F_{1}\left(1, m+\frac{1}{2} ; m+1 ; \frac{1}{1+c}\right) .
\end{aligned}
$$

Here, $c=b / 2 a, \Gamma(\cdot)$ is the gamma function such that $\Gamma(a)=\int_{0}^{\infty} e^{-t} t^{a-1} \mathrm{~d} t$, and ${ }_{2} F_{1}(\cdot, \cdot ; \cdot ; \cdot)$ is the Gaussian hypergeometric function. Substituting (11) and (12) into (10) yields the closed-form expression of BER as follows:

$$
\operatorname{BER}=M \sum_{k=0}^{M-1}(-1)^{k}\left(\begin{array}{c}
m-1 \\
k
\end{array}\right)\left(\frac{\sqrt{c / \pi}}{2(1+c)^{m+(1 / 2)}} \frac{\Gamma(m+(1 / 2))}{\Gamma(m+1)}{ }_{2} F_{1}\left(1, m+\frac{1}{2} ; m+1 ; \frac{1}{1+c}\right)\right)
$$

where $c=b / 2 a, a=n(k+1) / 4 \Omega S, b=2, m=1, n=M_{t}=$ $M_{\mathrm{r}}, M=M_{\mathrm{d}}$, the number of antennas at the destination. Equation (13) yields a closed-form expression for BER for the channel between the source and destination via the relay. Here, $\Omega$ and $S$ represent the average fading power and bit energy to noise spectral density, respectively. The average fading power is a function of power decay factor $(\Delta)$ such that $\Omega_{l}=e^{-(l-1) \Delta} \Delta \geq 0, l=1,2, \ldots, L, l$ is the number of the channel tap, and $\Omega_{l}$ is the average fading power of the $l_{\text {th }}$ propagation path.

4.1. Outage Probability $\left(P_{\text {out }}\right)$. The outage probability relative to $\gamma_{\text {th }}$ is defined as $P_{\text {out }} \equiv \int_{0}^{\gamma_{\text {th }}} p_{\gamma_{\text {srd }}}(\gamma) \mathrm{d} \gamma$. Therefore,

$$
P_{\text {out }}=\int_{0}^{\gamma_{\text {th }}} \sum_{k=0}^{M-1} M-1 \quad\left(-e^{((-\gamma n) / 4 \Omega S)(k+1)}\right) \frac{M n}{4 \Omega S} \mathrm{~d} \gamma
$$

To solve (14), the integral identity in Gradshteyn and Ryzhik ([21], equation (3.381.1)) is used as illustrated:

$$
\int_{0}^{u} x^{v-1} e^{-\mu x} \mathrm{~d} x=\mu^{-v} \Upsilon(v, \mu u)
$$

where $\Upsilon$ is the incomplete gamma function, defined as $\Upsilon(a, x)=\int_{0}^{x} t^{a-1} e^{-t} \mathrm{~d} t$. By substituting (15) into (14), we derive the closed-form expression for $P_{\text {out }}$ as follows:

$$
P_{\text {out }}=\sum_{k=0}^{M-1} M-1 \frac{M n}{4 \Omega S}\left(\frac{n(k+1)}{4 \Omega S}\right)^{-1} \Upsilon\left(\frac{n(k+1) \gamma_{\text {th }}}{4 \Omega S}, 1\right)
$$

4.2. Direct Link (without Relay). For the direct link between the source and destination, the closed-form expression for $P_{\text {out }}$ has been derived by Dixit and Katiyar in 2018 [17] and expressed as follows:

$$
P_{\text {out }}=\operatorname{gammainc}\left(\frac{\gamma_{\text {th }}}{\bar{\gamma}}, M_{t} M_{\mathrm{d}}\right) \text {, }
$$

where $M_{t} M_{\mathrm{d}}$ is the order of diversity, $\bar{\gamma}$ is the average fading power as described in (13), gammainc $(\cdot, \cdot)$ is the incomplete gamma function, and $\gamma_{\text {th }}$ is the threshold SNR. The closedform expression for BER is derived by Dixit and Katiyar [17] and expressed as 


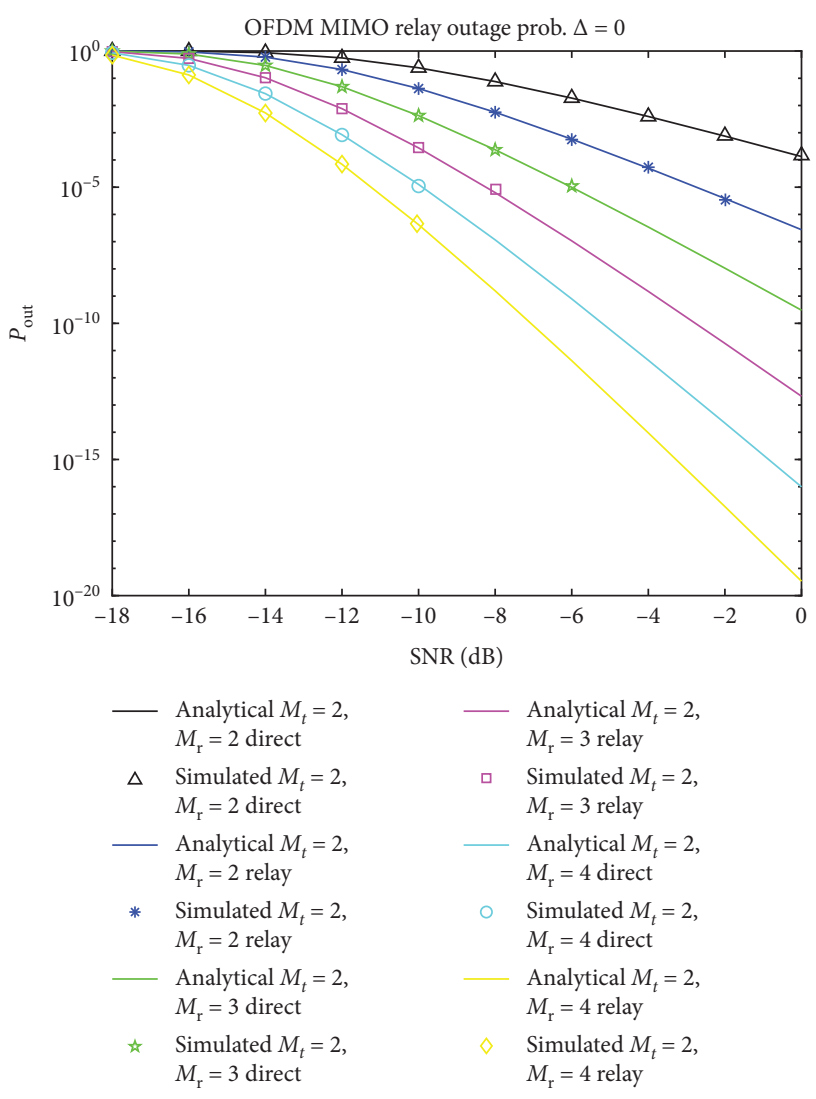

FIGURE 3: Outage probability with MIMO-OFDM direct and relay $(\Delta=0)$.

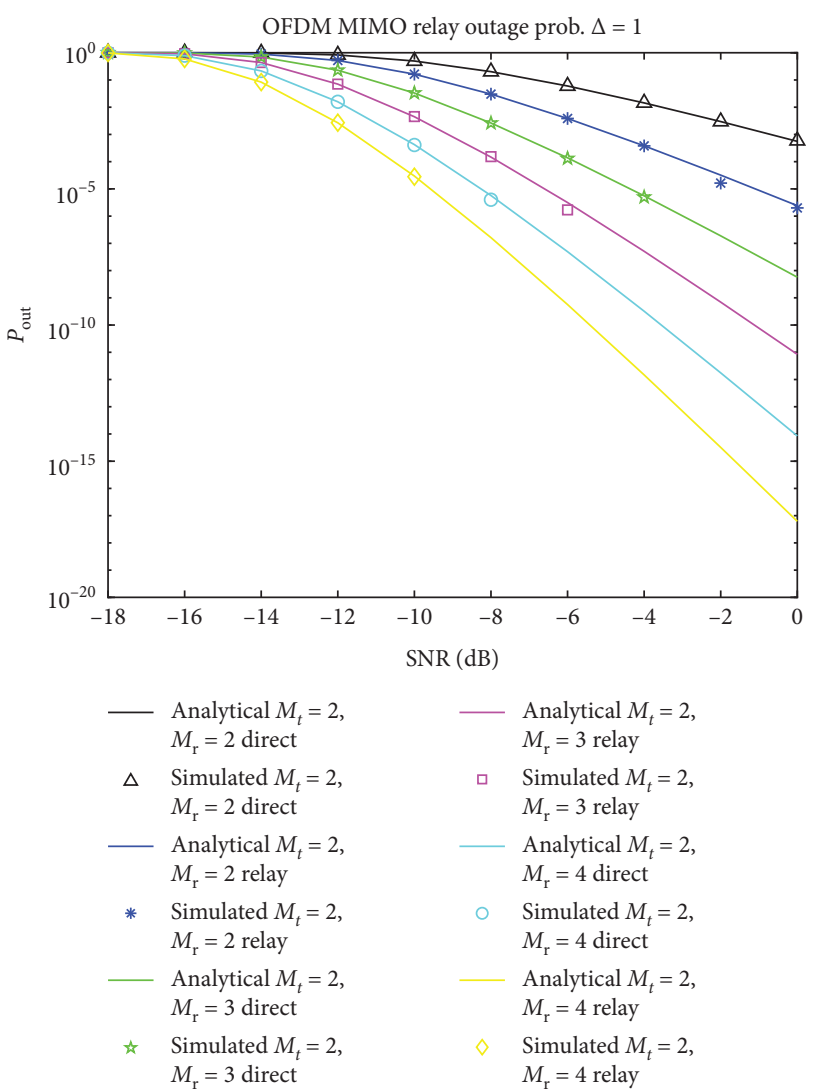

FIGURE 4: Outage probability with MIMO-OFDM direct and relay $(\Delta=1)$.

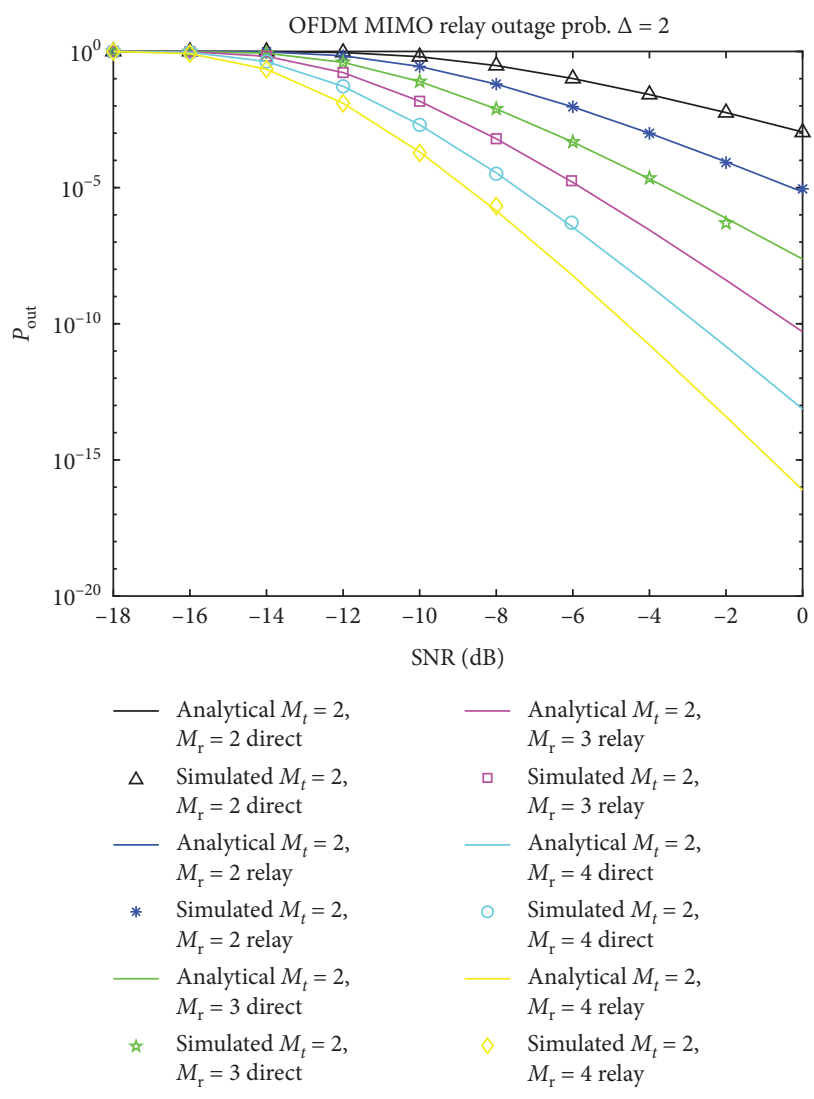

FIGURE 5: Outage probability with MIMO-OFDM direct and relay $(\Delta=2)$.

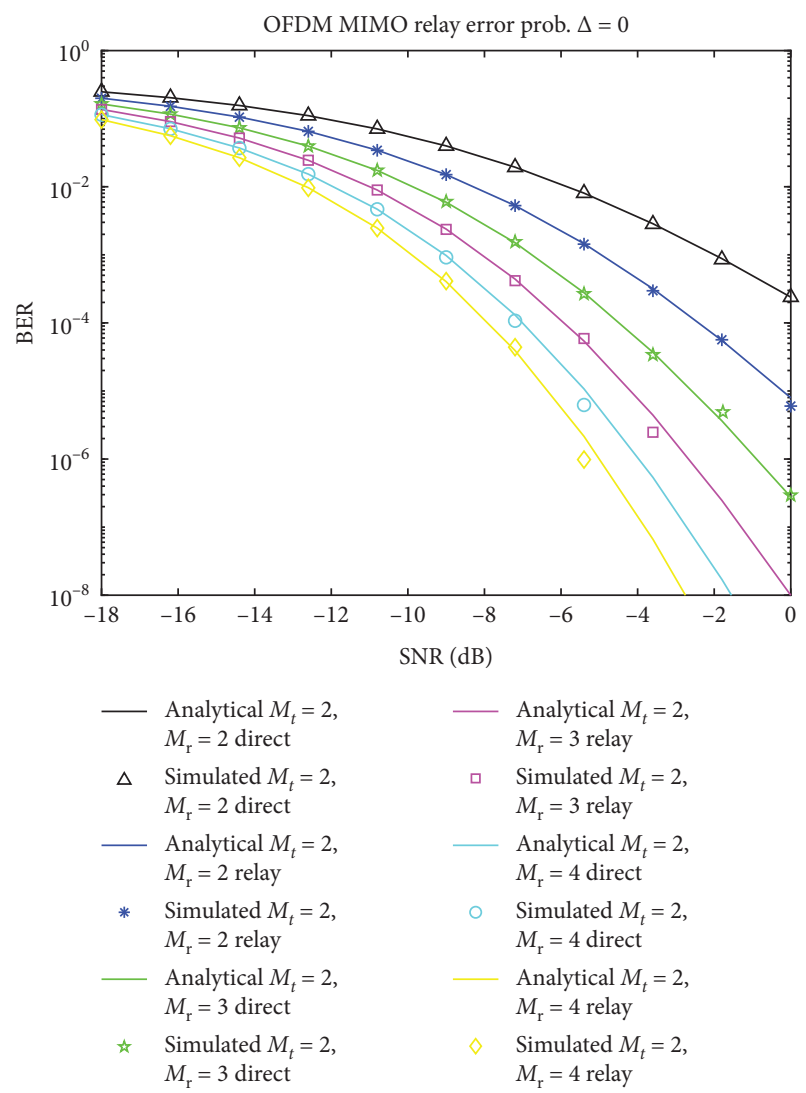

FIGURE 6: BER performance with MIMO-OFDM direct and relay $(\Delta=0)$. 


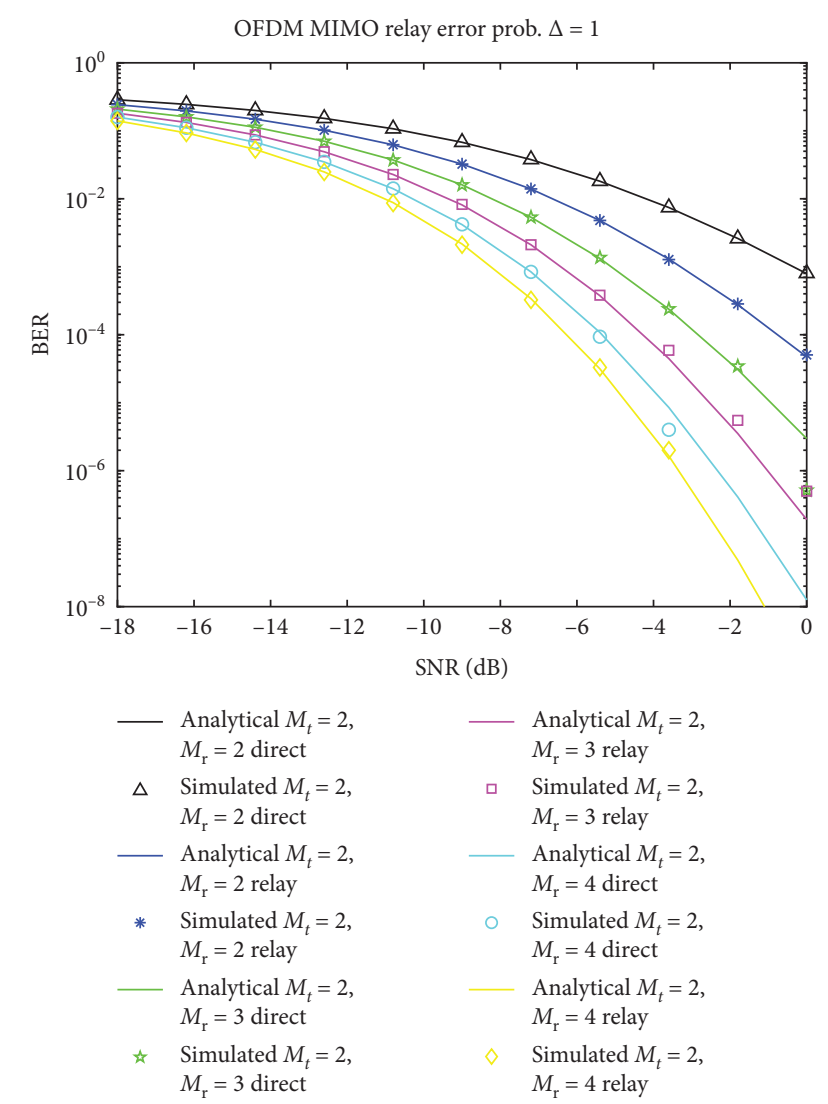

FIGURE 7: BER performance with MIMO-OFDM direct and relay $(\Delta=1)$.

$$
\begin{aligned}
\operatorname{BER}= & \frac{1}{a^{m}}\left(\frac{\sqrt{c / \pi}}{2(1+c)^{m+(1 / 2)}} \frac{\Gamma(m+(1 / 2))}{\Gamma(m+1)}\right. \\
& \left.\cdot{ }_{2} F_{1}\left(1, m+\frac{1}{2} ; m+1 ; \frac{1}{1+c}\right)\right),
\end{aligned}
$$

here $a=1 / 4 \Omega S, c=b / 2 a, m=M_{t} M_{\mathrm{d}}$, and $b=2$. In (17) and (18), $\bar{\gamma}$ and $\Omega$ represent the average fading power and are a function of power decay factor as described in (13).

\section{Simulation Results}

The closed-form expressions for BER and $P_{\text {out }}$ for the relay have been derived in (13) and (16). The closed-form expressions for BER and $P_{\text {out }}$ for the direct link are specified in (17) and (18), respectively. The results obtained from (13) and (16)-(18) are plotted and compared with the simulation values. The simulation results agree with the numerical results, thus validating our model.

Figure 3 plots the closed-form expression for outage probability for a decay factor of 0 . The simulated results closely match the numerical values. The performance of direct link is compared with that of relay link and the results demonstrate an improvement in the relay link. The relay performance improves with the higher number of antennas at the destination. Figures 4 and 5 plot the simulated and

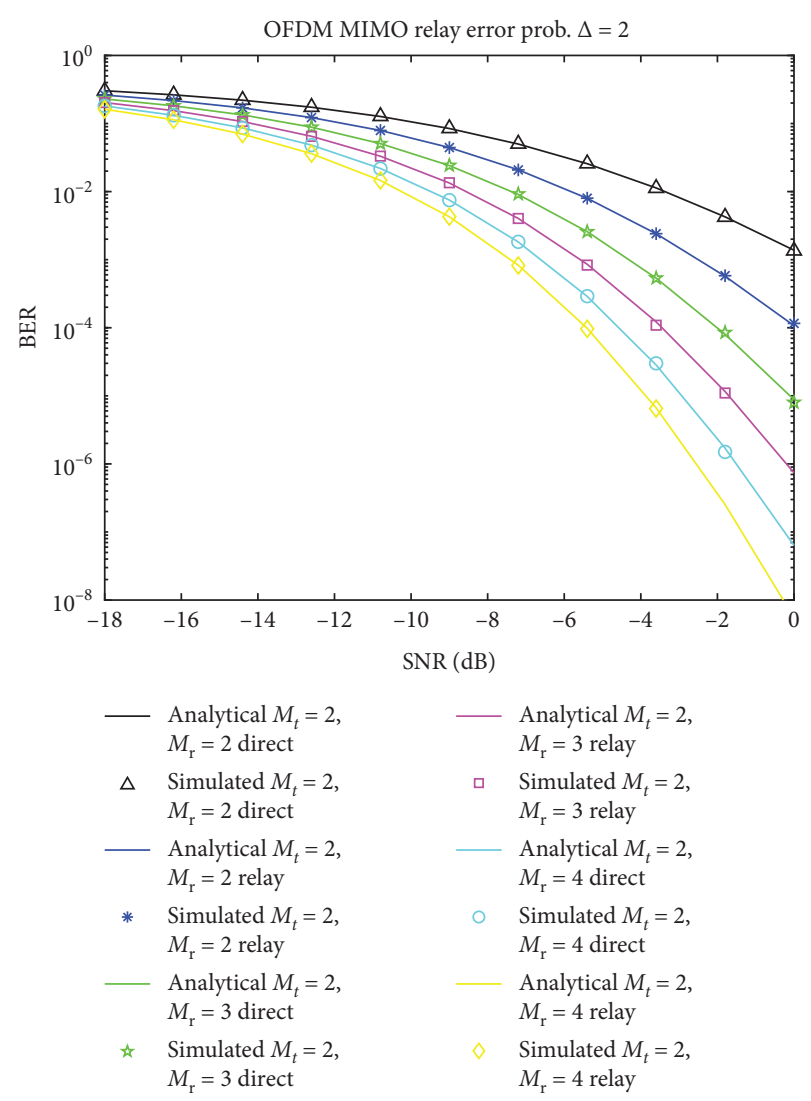

FIGURE 8: BER performance with MIMO-OFDM direct and relay $(\Delta=2)$.

closed-form expression values of outage probability for a power decay factor of 1 and 2, respectively. The number of transmit antennas at the source is taken as 2 , the same number of antennas at the relay, while, at the destination, 2/ 3/4 antennas combine.

Figure 6 plots the closed-form expression for BER for a decay factor of 0 . The simulated results closely match the numerical values. The performance of direct link is compared with that of relay link and the results demonstrate an improvement in the relay link. The relay performance improves with the higher number of antennas at the destination. Figures 7 and 8 plot the simulated and closed-form expression values of BER for a power decay factor of 1 and 2, respectively. The number of transmit antennas at the source is taken as 2 , the same number of antennas at the relay, while, at the destination, $2 / 3$ antennas combine.

\section{Conclusion}

The results demonstrate that cooperative relay communication offers performance improvement and combined with MIMO-OFDM provides an ISI free channel. The effect of power decay factor is also analyzed, and it is shown that high power decay leads to degradation in performance. The results in this paper are encouraging for advocating cooperative communications as a viable option, especially where the implementation of MIMO is constrained. 


\section{Data Availability}

The data used to support the findings of this study are included within the article.

\section{Conflicts of Interest}

The authors declare that they have no conflicts of interest.

\section{References}

[1] E. C. Van Der Meulen, "Three terminal communication channels," Advances in Applied Probability, vol. 3, no. 1, pp. 120-154, 1971.

[2] T. Cover and A. E. Gamal, "Capacity theorems for the relay channel," IEEE Transactions on Information Theory, vol. 25, no. 5 , pp. $572-584,1979$.

[3] V. Tarokh, H. Jafarkhani, and A. R. Calderbank, "Space-time block codes from orthogonal designs," IEEE Transactions on Information Theory, vol. 45, no. 5, pp. 1456-1467, 1999.

[4] P. Gupta and P. R. Kumar, "The capacity of wireless networks," IEEE Transactions on Information Theory, vol. 46, no. 2 , pp. $388-404,2000$

[5] A. Sendonaris, E. Erkip, and B. Aazhang, "User cooperation diversity-part II: implementation aspects and performance analysis," IEEE Transactions on Communications, vol. 51, no. 11, pp. 1939-1948, 2003.

[6] A. Nosratinia, T. E. Hunter, and A. Hedayat, "Cooperative communication in wireless networks," IEEE Communications Magazine, vol. 42, no. 10, pp. 74-80, 2004.

[7] M. Dohler, A. Gkelias, and H. Aghvami, "2-hop distributed MIMO communication system," Electronics Letters, vol. 39, no. 18 , pp. 1350-1351, 2003.

[8] J. N. Laneman, D. N. C. Tse, and G. W. Wornell, "Cooperative diversity in wireless networks: efficient protocols and outage behavior," IEEE Transactions on Information Theory, vol. 50, no. 12, pp. 3062-3080, 2004.

[9] S. Cui, A. J. Goldsmith, and A. Bahai, "Energy-efficiency of MIMO and cooperative MIMO techniques in sensor networks," IEEE Journal on Selected Areas in Communications, vol. 22, no. 6, pp. 1089-1098, 2004.

[10] O. S. Shin, A. Chan, H. T. Kung, and V. Tarokh, "Design of an OFDM co-operative space- time diversity system," IEEE Transactions on Vehicular Technology, vol. 56, no. 4, pp. 2203-2215, 2007.

[11] A. Tolli, M. Codreanu, and M. Juntti, "Cooperative MIMOOFDM cellular system with soft handover between distributed base station antennas," IEEE Transactions on Wireless Communications, vol. 7, no. 4, pp. 1428-1440, 2008.

[12] M.-s. Baek and H.-k. Song, "Cooperative diversity technique for MIMO-OFDM uplink in wireless interactive broadcasting," IEEE Transactions on Consumer Electronics, vol. 54, no. 4, pp. 1627-1634, 2008.

[13] Y. Jing and H. Jafarkhani, "Single and multiple relay selection schemes and their achievable diversity orders," IEEE Transactions on Wireless Communications, vol. 8, no. 3, pp. 14141423, 2009.

[14] H. Lu, H. Nikookar, and T. Xu, OFDM Communication with Cooperative Relays, Communications and Networking, Intech Publisher, Rijeka, Croatia, 2010.

[15] M. O. Hasna and M.-S. Alouini, "Outage probability of multihop transmission over nakagami fading channels," IEEE Communications Letters, vol. 7, no. 5, pp. 216-218, 2003.
[16] B. Chalise and L. Vandendorpe, "Outage probability analysis of a MIMO relay channel with orthogonal space-time block codes," IEEE Communications Letters, vol. 12, no. 4, pp. 280-282, 2008.

[17] S. Dixit and H. Katiyar, "OFDM for $4 \mathrm{G}$ wireless communications," International Journal of Interdisciplinary Telecommunications and Networking, vol. 10, no. 1, pp. 63-84, 2018.

[18] A. Papoulis and S. U. Pillai, Probability, Random Variables, and Stochastic Processes, Tata McGraw Hill, New Delhi, India, 2002.

[19] A. Goldsmith, Wireless Communications, Cambridge University Press, New York, NY, USA, 2005.

[20] M. K. Simon and M.-S. Alouini, Digital Communication over Fading Channels, John Wiley and Sons, Hoboken, NJ, USA, 2005.

[21] I. S. Gradshteyn and I. M. Ryzhik, Table of Integrals, Series and Products, Academic Press, San Diego, CA, USA, 7th edition, 2007. 


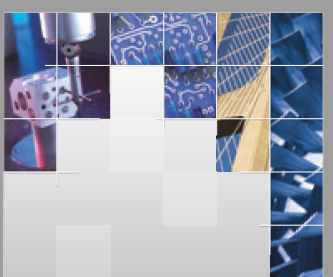

\section{Enfincering}
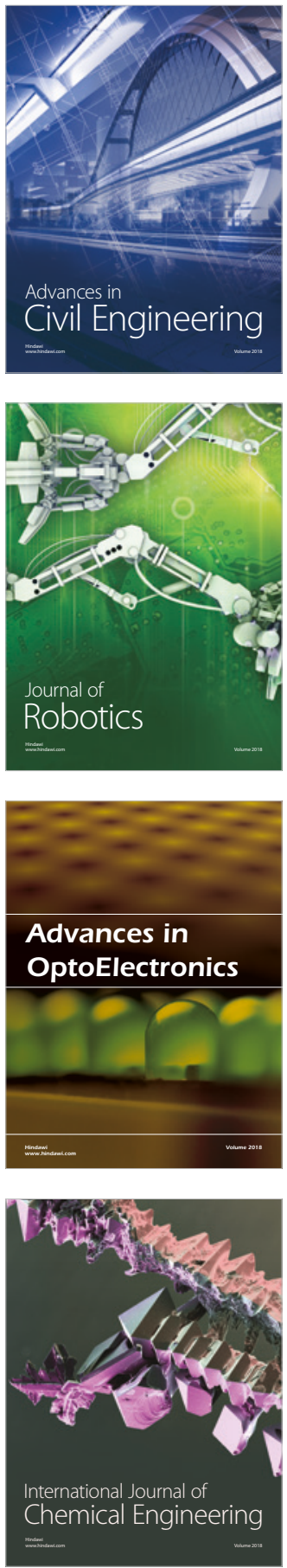

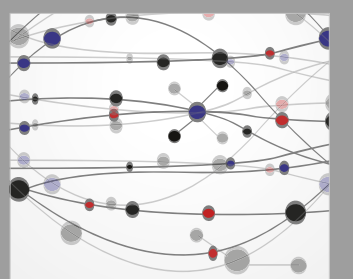

\section{Rotating \\ Machinery}

The Scientific World Journal

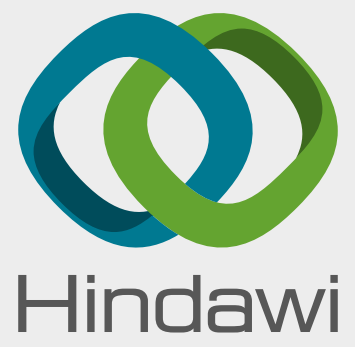

Submit your manuscripts at

www.hindawi.com
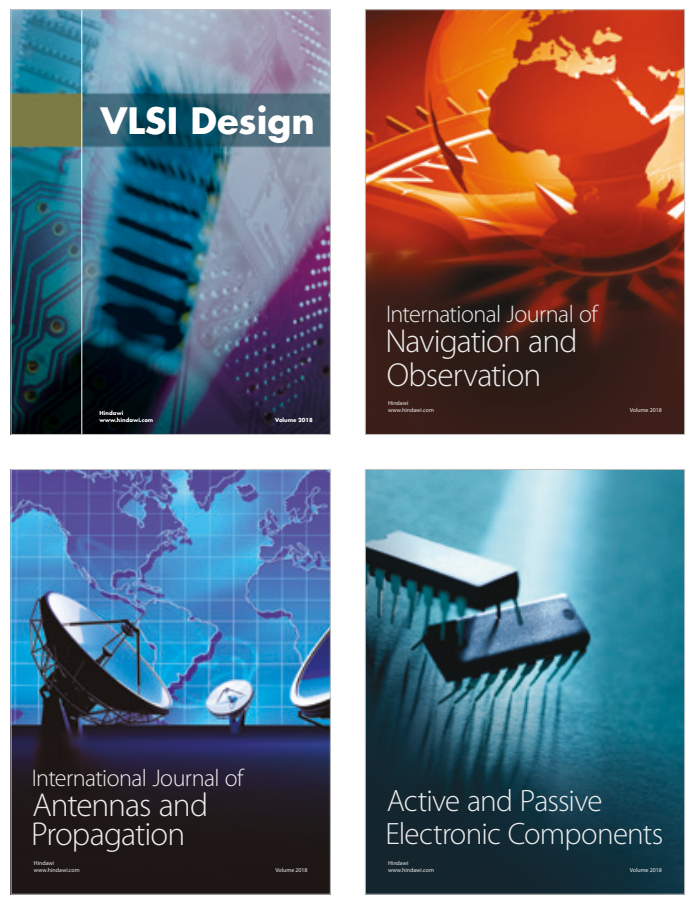
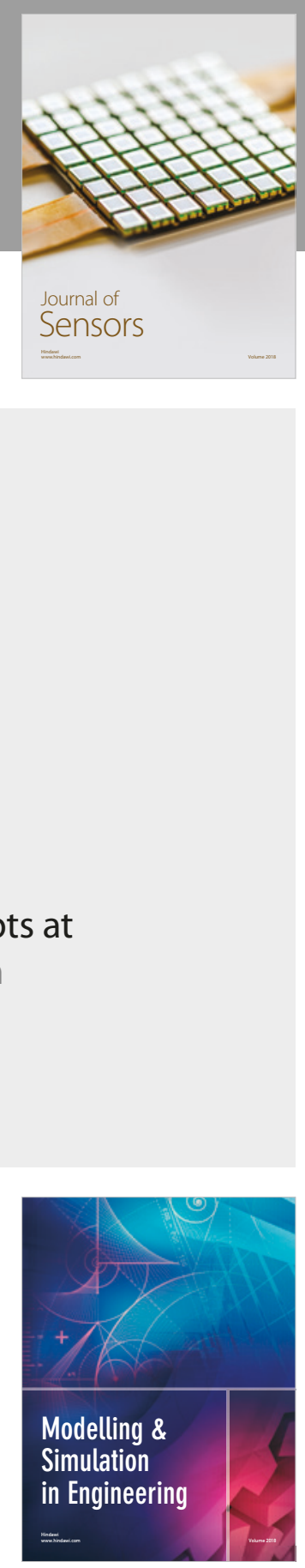

\section{Advances \\ Multimedia}
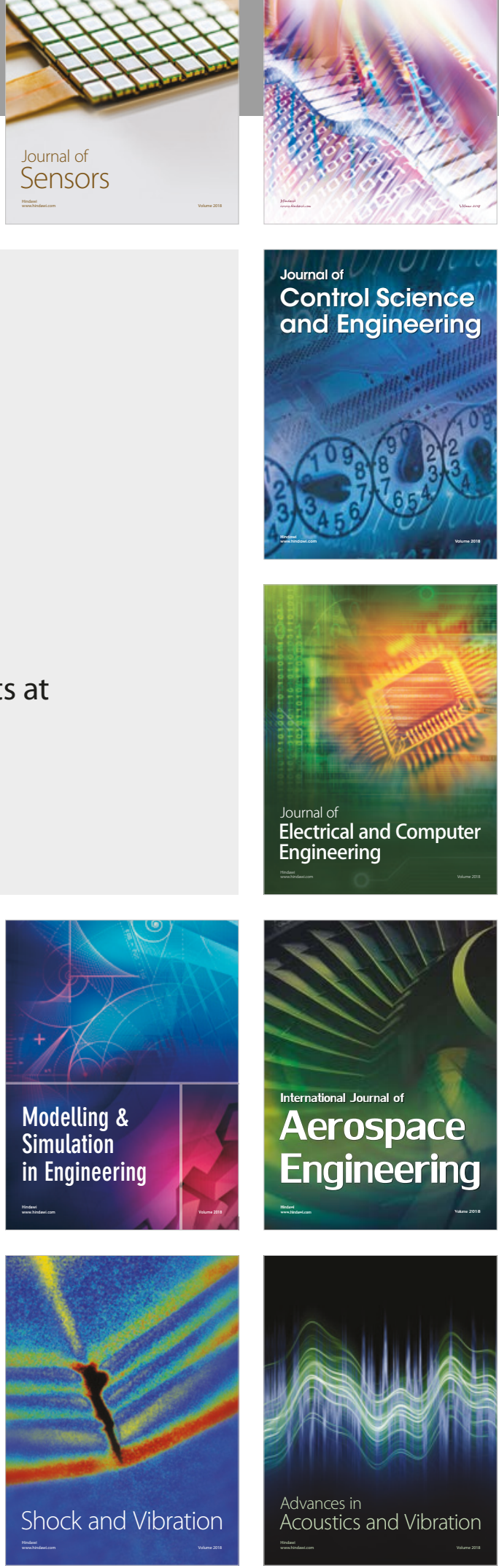